\title{
Scanning electron microscopy and molecular dynamics of surfaces of growing and ablating hexagonal ice crystals
}

\author{
W. C. Pfalzgraff, R. M. Hulscher, and S. P. Neshyba \\ University of Puget Sound, Tacoma, Washington, 98416, USA \\ Received: 10 August 2009 - Published in Atmos. Chem. Phys. Discuss.: 1 October 2009 \\ Revised: 19 March 2010 - Accepted: 23 March 2010 - Published: 29 March 2010
}

\begin{abstract}
We present the first clearly resolved observations of surfaces of growing and ablating hexagonal ice crystals using variable-pressure scanning electron microscopy. The ice surface develops trans-prismatic strands, separated from one another by distances of $5-10 \mu \mathrm{m}$. The strands are present at a wide range of supersaturations, but are most pronounced at temperatures near the frost point. Pyramidal facets consistent with Miller-Bravais indices of $10 \overline{1} 1$, and possibly also $20 \overline{2} 1$, are associated with ice growth under these conditions. A molecular-dynamics model of a free-standing ice $I_{h}$ nanocolumn containing 8400 water molecules does not develop trans-prismatic strands, suggesting these features originate at larger spatial or temporal scales. The possible relevance of these surface features to cirrus ice is discussed.
\end{abstract}

\section{Introduction}

One objective of cloud microphysics is to represent the morphology of cirrus cloud crystals well enough that their optical properties are faithfully represented in climate models (Stephens et al., 1990). On a spatial scale of $10-100 \mu \mathrm{m}$, this objective has been met with considerable success; a great diversity of crystal habits has been recorded, including bullets, bullet rosettes, hollows, aggregates, and droxtals (e.g., Yang et al., 2005; Bailey and Hallett, 2004). We know, for example, that ice crystals in midlattitude cirrus clouds have a preponderance of relatively spherical (although still faceted) shapes near the top, and hollow hexagonal prisms at midlevel (Heymsfield and Iaquinta, 2000). Laboratory studies have established patterns of dependency of crystal habit on temperature, supersaturation, and vapor diffusivity, among other factors (e.g., Bailey and Hallett, 2004; Libbrecht, 2003), so

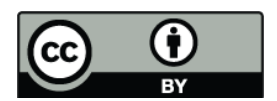

Correspondence to: S. P. Neshyba (nesh@pugetsound.edu) that the dynamics of habit evolution may, in principle, be included in cloud-resolving models. Radiative transfer methods, moreover, allow one to predict how these varying shapes affect the optical properties of cloud particles (Neshyba et al., 2003; Yang et al., 2008a).

Dynamics of morphological properties of ice on a mesoscopic (micrometer-level) scale are less well characterized. Optical micrographs of falling atmospheric ice crystals originating under cirrus-like conditions exhibit mesoscopic texturing of various kinds, including step formation on the inside of hollow hexagonal prisms, and corrugations on the outside (Walden et al., 2003; Yang et al., 2008a); micrographs of laboratory-grown ice crystals exhibit a similarly large array of surface structures (e.g., Hallett and Mason, 1958). Limitations inherent to optical microscopy, however, place definite limits on one's ability to discern details of such structures, and relatively little attention has been paid to how those structures evolve over the time scale pertinent to cirrus cloud formation and dissipation.

Evidence for radiometrically significant mesoscopic structure derives from in situ studies of the light-scattering properties cirrus clouds, which have revealed a distribution of asymmetry parameters whose mean is smaller than can be accounted for by crystal habit alone (Gerber et al., 2000; Lampert et al., 2009). To account for the discrepancy, statistical representations of mesoscopic roughness have been invoked (Garrett, 2008). This modification works in the desired direction (to lower asymmetry parameters) (Yang and Liou, 1998; Ulanowski et al., 2006), but now a new set of questions arises: In what sense are the surfaces of cirrus ice crystals mesoscopically rough, and how does this roughness evolve over time? Does the roughness depend on the same factors known to determine crystal habit, and if so, then how?

In principle, theories of ice crystal growth and ablation could help settle questions regarding the morphology and dynamics of mesoscopic structure. Some ice growth models invoke assumptions about mesoscopic surface structure

Published by Copernicus Publications on behalf of the European Geosciences Union. 
as an integral part of the growth mechanism (Gravner and Griffeath, 2009; Wood et al., 2001), but these theories do not predict the scale of such roughness, which is essential information for optical scattering purposes. Molecular dynamics (MD) may eventually provide insight into the molecularlevel processes connected to surface morphology, provided the relevant time and spatial scales are achievable; simulation of a few thousand water molecules over a few milliseconds can take weeks of computer time.

Scanning electron microscopy (SEM) has long been recognized as an attractive alternative for observing ice morphology (Cross, 1968; Rango et al., 2000; Erbe et al., 2003), because of its resolution and depth of field. However, the high vacuum conditions and coating procedures usually employed to prepare samples do not permit observation of surface dynamics. Recently, however, variable pressure SEM (VP-SEM) has been used to study crystals grown inside the chamber of the instrument and imaged as they grew (Zimmerman et al., 2007; Cartwright et al., 2008). Surface features have remained largely unexplored with this technique because the crystals grown were small (tens of micrometers in diameter), and the increase in current density for magnifications above 1000x caused melting or evaporation of the ice.

Here, we present VP-SEM micrographs showing surface features associated with growth and ablation of hexagonal ice prisms growing on a cold copper substrate. We observe the growth of hexagonal ice crystals that are several hundred micrometers in diameter. Subsequent manipulations allow us to examine the surfaces of these crystals at near-zero supersaturation (both growth and ablation conditions) at magnifications of 400-700x. A limitation of this approach is that the maximum pressure inside commercial VP-SEMs, tens to hundreds of Pascals, is much lower than conditions met by real cirrus ice crystals. Therefore, the relevance of morphology of a vapor-deposited ice surface to real cirrus cloud crystals must be carefully evaluated; these considerations are discussed in more detail below.

We also present results of molecular dynamics (MD) simulations of a free-floating hexagonal ice prism, undertaken in the hope that atomistic counterparts to observed structures may provide insight into their microscopic origins.

\section{Methods}

\subsection{Imaging growing and ablating ice hexagons in a VP- SEM chamber}

Micrographs were obtained using a Hitachi S-3400N VPSEM equipped with a backscattered electron detector and an accelerating voltage of $10-15 \mathrm{kV}$. A rough-cut copper specimen stub mounted on an examination stage was cooled with a Deben Ultra Coolstage MK3 version Peltier cooling element. Water vapor was introduced by placing a metal reser- voir with $\sim 4 \mathrm{~mL}$ of de-ionized liquid water inside the VPSEM chamber. Upon switching on the evacuation pump, the reservoir water cooled evaporatively. Once an operating pressure of $50 \mathrm{~Pa}$ was reached, the Peltier cooling system was set to the desired temperature for the experiment (between -30 and $\left.-45^{\circ} \mathrm{C}\right)$. The examination stage was then monitored at low magnification $(\sim 20 \times)$ until the first ice crystals were evident.

Once ice crystals were detected, two types of experimental procedure were used; both were designed to examine surface morphological changes under conditions close to zero supersaturation. In an isothermal experiment, the temperature of the examination stage was held constant (usually $-45^{\circ} \mathrm{C}$ ). During this time, the partial pressure of water vapor in the SEM chamber decreased slowly, eventually falling below frost point of the examination stage. This process typically takes about one hour (depending on the amount of water vapor initially in the reservoir). In an ablation experiment, the temperature of the examination stage was quickly raised above the frost point temperature, a process requiring at most one minute. Either way, surface morphology changes during a transition from supersaturated to subsaturated could be studied. Quantitative aspects of these procedures are discussed in the next section.

\subsection{Molecular dynamics of a free-standing nanocolumn and a prismatic slab}

A rectangular volume of ice $I_{h}$, comprised of thirty ice bilayers of 280 molecules each (totaling 8400 molecules), was initially constructed according to the proton disordering algorithm of Buch et al. (1998). The orientation of this volume was such that the basal surface lay perpendicular to the $\mathrm{z}$-axis, and the prismatic surface lay perpendicular to the $\mathrm{y}$ axis. Initial dimensions of the crystal were 4.46, 5.45, and $11.00 \mathrm{~nm}$ in the $\mathrm{x}, \mathrm{y}$, and $\mathrm{z}$ directions. For the first part of the MD simulation, box dimensions were extended in the $\mathrm{x}$ and y dimensions to 7.5 and $8.0 \mathrm{~nm}$, respectively, for $20 \mathrm{ns,}$ to allow the system to anneal to an approximately hexagonal cross section (after Pereyra and Carignano, 2009). Subsequently, the simulation box in the $\mathrm{z}$ direction was extended to $14.4 \mathrm{~nm}$, thereby exposing the basal surfaces to vacuum as well. Integration of classical equations of motion was performed by Gromacs molecular dynamics software (Lindahl et al., 2001), with rigid molecules interacting according to a 6-site intermolecular potential. (Nada and van der Eerden, 2003), constant NVT conditions, a 1-femtosecond integration step, Berendsen temperature coupling with a time constant of $0.1 \mathrm{ps}$, and a temperature of $-23^{\circ} \mathrm{C}$. For long-range Coulomb interactions, particle-mesh Ewald summation was used with a relative tolerance of $1 \times 10^{-5}$, 4 th order cubic interpolation, and a Fourier spacing parameter of 0.12. For Van der Waals interactions, a cut-off radius of $0.07 \mathrm{~nm}$ was used. Angular and linear momentum of the system as a whole were removed at intervals of 50 femtoseconds. This system is 


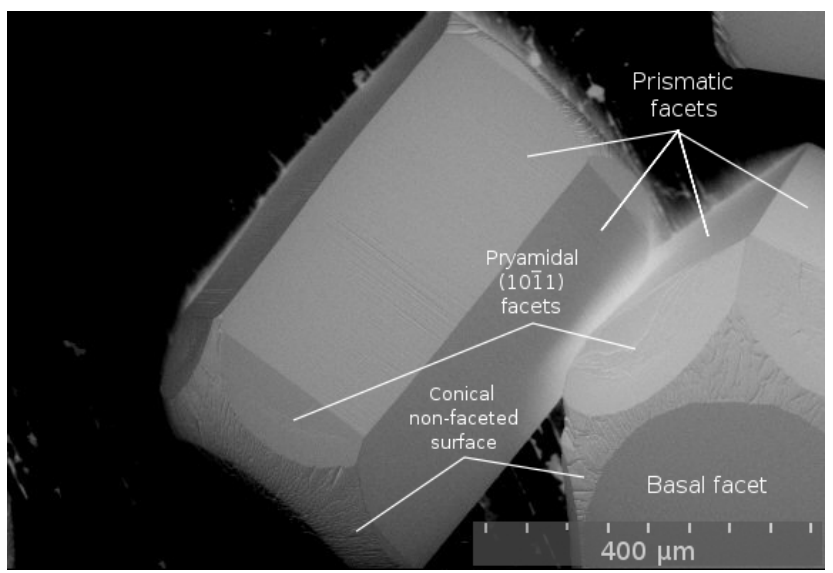

Fig. 1. VP-SEM images of growing ice crystals. The black background is the copper substrate.

referred to hereinafter as a free-standing nanocolumn. At the simulation temperature considered here $\left(-23^{\circ} \mathrm{C}\right)$, ice surfaces exposed to vacuum develop a quasi-liquid layer. For the purpose of visualizing only the underlying ice, quasi-liquid molecules were defined as those with an average of 3.8 or fewer hydrogen bonds over a $1 \mathrm{~ns}$ period; this criterion identifies slightly more molecules as quasiliquid than the condition used by Carignano et al. (2005), who used a cutoff value of 3.3 hydrogen bonds. VMD imaging software was used to visualize simulation results in such a way that quasiliquid molecules were rendered differently from underlying ice (Humphrey et al., 1996).

\section{Results}

\subsection{SEM images}

Crystals were first visible on the examination stage typically 5-15 min after the beginning of an SEM session. Of the visible crystals, $20-50 \%$ were isolated hexagonal prisms, the rest polycrystalline aggregates. In the first few minutes of growth, crystals usually grew too quickly to produce highresolution images, although they could be monitored in real time at low resolution.

Figure 1, taken 12 min after initial ice formation was observed, shows two hexagonal crystals. The crystal on the left is more typical in that its prismatic surface is visible at a near-normal angle, and its basal facet is tilted away from the detector. This preference does not appear to be a function of impurities on the copper substrate. The crystal has beveled facets associated with each prismatic surface, designated pyramidal facets. A second crystal in Fig. 1 is atypical in that the basal facet faces the detector. During early, fast growth, crystals also typically displayed a non-faceted surface with conical symmetry as well. This surface is small or not present in larger, slower-growing crystals.

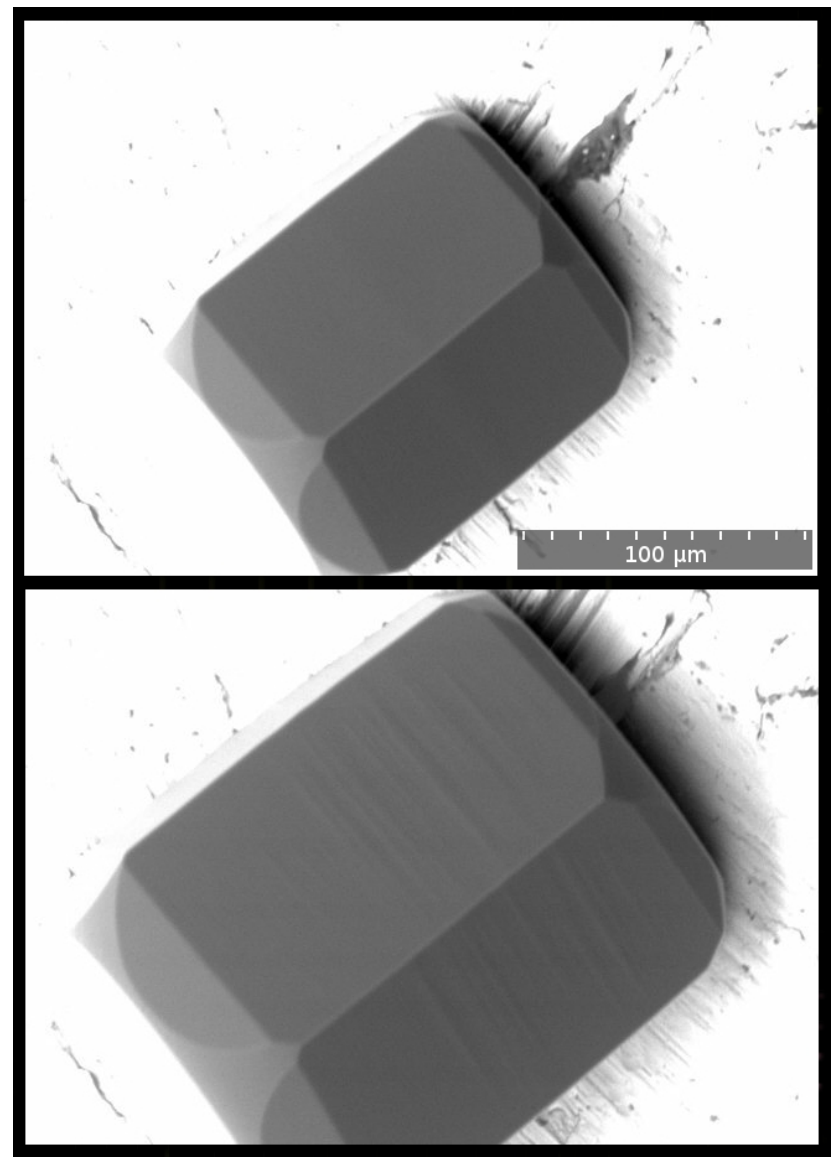

Fig. 2. Hexagonal ice crystal in an early stage of growth. The first micrograph was taken seconds after the crystal was noticed, the second was taken $32 \mathrm{~s}$ later.

Figure 2 shows an isolated hexagonal crystal at an early growth stage. The first micrograph in the series was taken immediately after nucleation on the copper specimen stub. The second micrograph was taken 32 seconds later, implying growth rates of 0.7 and $0.8 \mu \mathrm{m} \mathrm{s}^{-1}$ in the prismatic and basal facets, respectively.

Figure 3 shows a view of a crystal during an isothermal experiment at $-45^{\circ} \mathrm{C}$. As described in Sect. 2, the vapor pressure of water during any given VP-SEM session declines over the course of about an hour. In this session, the transition from supersaturation conditions to subsaturation conditions (the "ablation transition") was marked by the change in surface morphology evident between Fig. 3a and b. Micrographs shown to the right of Fig. $3 a$ and $b$ are a series of higher-magnification micrographs, taken at $\sim 30$ s intervals before and after the ablation transition. Before continuing with an examination of these micrographs, however, it is useful to consider how the morphological changes evident in Fig. 3a and $\mathrm{b}$ can be used to estimate the supersaturation inside the VP-SEM chamber. 

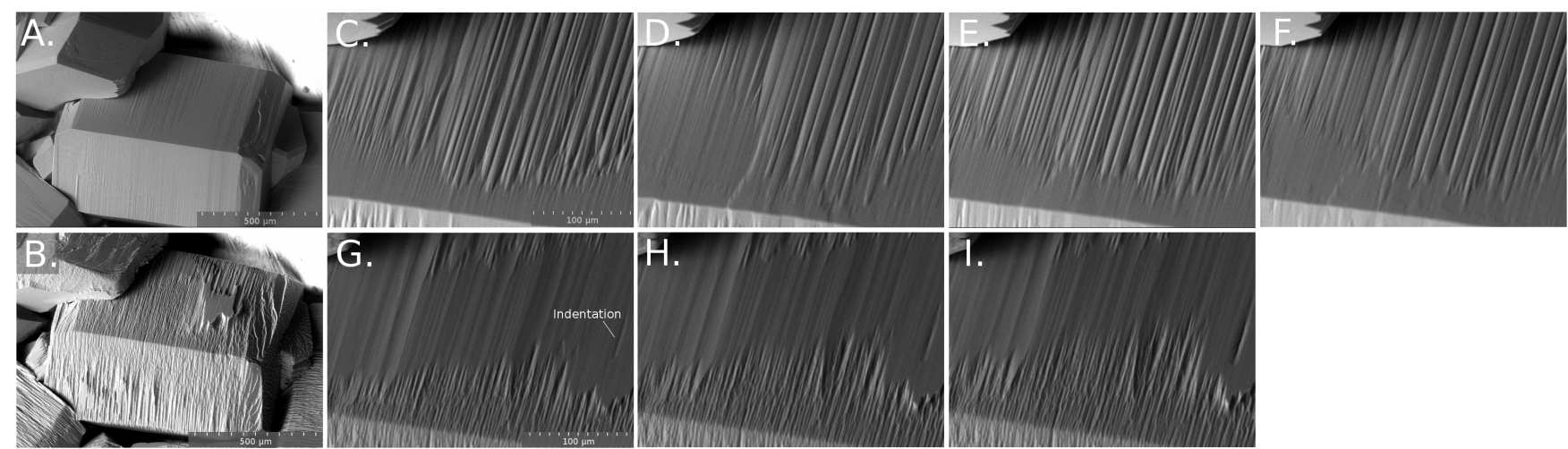

Fig. 3. Growth and ablation sequences. (A) Low magnification view of a growing ice crystal. (B) View of the same crystal after $\sim 15$ min of ablation conditions. (C-F) Higher magnification sequence showing growth at the prismatic edge at the center of A, taken at intervals of $\sim 30$ s. (G-I) Higher magnification sequence showing ablation at the same prismatic edge, also at intervals of $\sim 30 \mathrm{~s}$.

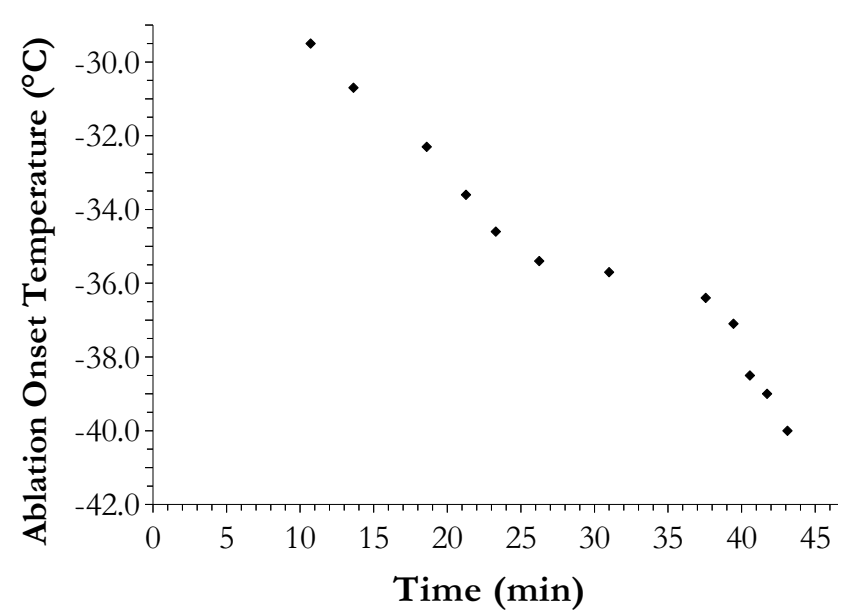

Fig. 4. Ablation onset temperature as a function of time for a typical VP-SEM session.

We note first that similar morphological transitions can be induced in an ablation experiment. Cycles of raising and lowering the temperature over the course of a single VP-SEM session can thereby produce a record of the evolving ablation transition temperature for the session. We assume that these transition temperatures define a facet frost point temperature, which in turn determines supersaturation for a given isotherm. Numerous such experiments were conducted in an effort to characterize a typical VP-SEM session; Fig. 4 shows a typical result. The precipitous decline after $35 \mathrm{~min}$ occurs because the water reservoir has run dry.

Figure 5 shows the supersaturation at the examination stage for the above-mentioned "typical" case, obtained by

$\sigma=\left(\frac{P_{\text {vap }}\left(T_{f}\right)}{P_{\text {vap }}\left(T_{S}\right)}-1\right) \times 100$ where $T_{f}$ is the ablation onset temperature taken from Fig. 4 , $T_{S}$ is the temperature of the examination stage, and $P_{\text {vap }}$ is given by Buck (1981). Values begin at $10 \mathrm{~min}$, when the first crystals appeared during this VP-SEM session. Under the assumption that this VP-SEM session represents a typical case, Fig. 5 can be used to infer approximate supersaturation for other sessions, by noting the time before or after the ablation transition a given micrograph was taken. Values past $43 \mathrm{~min}$ were not possible to obtain in this session because of the precipitous decline in ambient water vapor noted above; the extension of the $-45^{\circ} \mathrm{C}$ isotherm to $50 \mathrm{~min}$ in Fig. 5 is an extrapolation.

As an example, if the isothermal experiment of Fig. 3 were typical, its trajectory would correspond to the $-45^{\circ} \mathrm{C}$ contour of Fig. 5. The timing of the last micrograph taken under growth conditions in the series (3F) relative the ablation transition is indicated in Fig. 5, implying a supersaturation of $25 \%$. Likewise, the timing of the first micrograph taken under ablation condition in the second series $(3 \mathrm{G})$ implies a supersaturation of $-25 \%$.

Turning next to dynamics, it will be useful to distinguish the strands that appear in Figs. 3C-F as "growth strands", and those that appear in Figs. 3G-I as "ablation strands." Growth strands are typically separated from one another by $5-10 \mu \mathrm{m}$. Supplemented by real-time observations (not shown), the sequence $3 \mathrm{C}-\mathrm{F}$ suggests collective dynamics: originating near one prismatic edge, strand-ends often advanced together, forming a front; once the front came within a few tens of micrometers from the adjacent edge, it retreated, leaving a smooth surface immediately adjacent to the prismatic edge. This collective advance and retreat continued at irregular intervals of 20-40 s.

The ablation strands appearing in Figs. 3G-I, also taken at $\sim 30 \mathrm{~s}$ intervals, but under ablation conditions, appear to deepen first at the prismatic edge, and at indentations in the smooth prismatic surface away from the edge. The deepening extends along the prismatic strands, eventually 


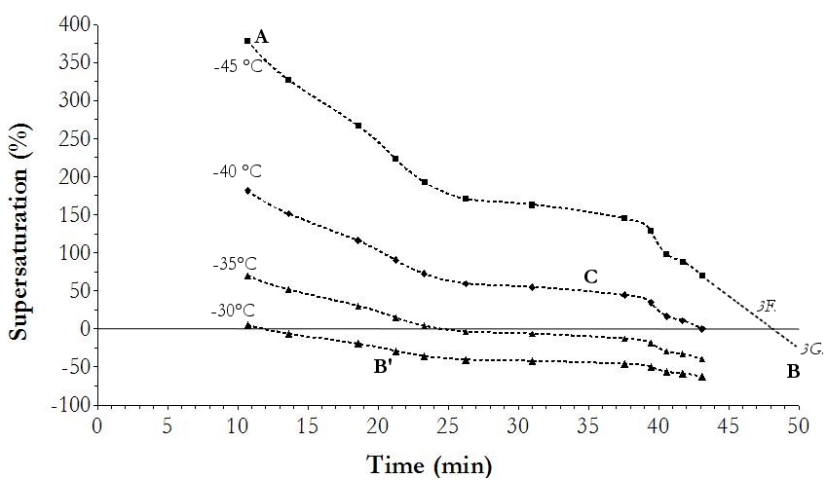

Fig. 5. Supersaturation values at the examination stage for a typical VP-SEM session for nominal stage temperatures ( $T_{s}$ in Eq. 1$)$. Ablation onset temperatures ( $T_{f}$ in Eq. 1$)$ are taken from Fig. 4 . The $-45^{\circ} \mathrm{C}$ curve is extrapolated to point $\mathrm{B}$, since at that point in the session the examination stage could not be cooled enough to induce another growth transition.

roughening nearly the entire prismatic surface. The result is that, unlike growth strands, ablation strands are roughest and deepest near the prismatic edge, and the overall texture of the ablated surface is rougher as well. Finally, it is striking that the prismatic edge remains clearly defined despite pronounced ablation.

On the pyramidal facets shown in Fig. 3, ledges and terraces are evident under both growth and ablation conditions, suggesting that growth and ablation on these facets are not mediated by trans-prismatic strands but instead by the formation and ablation of ledges.

We turn next to the question of how previously ablated crystals recover when re-exposed to growth conditions. Figure $6 \mathrm{a}-\mathrm{d}$ show an ablated crystal near a prismatic edge at $600 \mathrm{x}$ magnification, after returning the temperature of the examination stage to $-45^{\circ} \mathrm{C}$. The first visual cue that re-growth is occurring is the appearance of a short-lived, smooth surface along the remains of the prismatic edge (Fig. 6b), which quickly spreads to cover the rough texture along the ablated edge. Within minutes, these smooth surfaces appear to define beveled precursor segments to a reforming prismatic edge, several of which are visible in Fig. 6c and d. Figure 6e$\mathrm{h}$, taken approximately $90 \mathrm{~s}$ apart, demonstrate continued regrowth. The reconstruction of the smooth surface proceeds in a zipper-like fashion, moving up and to the right in the figure. We also note that the ablated area changes very little in this sequence. The set of Fig. 6i-l is a sequence of higher magnification views taken a few minutes later, still under re-growth conditions. The similarity between the structure and dynamics of this re-growing prismatic surface and those of Fig. $3 \mathrm{c}-\mathrm{e}$ suggests identical growth mechanisms. This sequence further demonstrates that strand-mediated growth is not operative on other facet types; the pyramidal facet appears to regrow by the formation of much larger ledges $(20 \mu \mathrm{m}$ wide and $5 \mu \mathrm{m}$ deep).
An analysis of the angle subtended by pyramidal facets with respect to the prismatic plane, as a function of the shoulder ratio appearing to the detector, is shown in Fig. 7. These angles are separated into two groups, one clustered around $28^{\circ}$ (Miller-Bravais indices, 1011), and a second, less common, set clustered around $14^{\circ}$ (Miller-Bravais indices, 20ㄹ1). The $\Theta$-intercept, corresponding to a shoulder ratio of zero, has a standard deviation of $\pm 1^{\circ}$ for both plots.

\subsection{Molecular dynamics simulations}

Figure 8 shows the integrated oxygen atom number density of the free-standing nanocolumn as a function of distance along the crystallographic c-axis, for various times after exposure of basal surfaces to vacuum. Each curve was obtained by integrating over $0.2 \mathrm{~ns}$. The changes between $0-5 \mathrm{~ns}$ constitute movement of approximately 100 molecules past the original terminus of the model (i.e., $z>12 \mathrm{~nm}$ in the figure). This movement slows considerably, resulting in no appreciable change in density from 5 to $8 \mathrm{~ns}$. Longer trajectories to $100 \mathrm{~ns}$ (not shown here) indicate a continuing, relatively slow thickening of the nanocolumn center, but no indication of melting (for example, bilayer integrity remains intact). The inset shows the potential energy of the system over this interval; it has a standard deviation of $0.06 \mathrm{~kJ} / \mathrm{mol}$, with a mean of $-48.1 \mathrm{~kJ} / \mathrm{mol}$.

Figure 9 shows a configuration of the free-standing nanocolumn $4 \mathrm{~ns}$ after the exposure of basal surfaces to vacuum, with ice-like molecules rendered as solid shapes and quasi-liquid molecules rendered semi-transparently. Basal and prismatic facets are evident. Also evident are pyramidal facets similar to those appearing in the VP-SEM images. Analysis of these surfaces indicates that these facets subtend an angle of $28^{\circ}$ with respect to adjacent prismatic facets, consistent with Miller-Bravais indices (1011). Figure 9b shows that a prismatic edge appears to be forming.

Finally, we see in the MD simulations no clear evidence of growth instability identifiable as trans-prismatic strands. Indeed, underneath the quasi-liquid layer the basal and prismatic facets have nearly perfect molecular smoothness. One possible inference from this observation is that trans-prismatic strands originate at a higher level of spatial. $(>10 \mathrm{~nm})$ or temporal organization.

\section{Discussion}

The predominant pyramidal facet observed here with VPSEM, subtending an angle of $28^{\circ}$ with respect to the prismatic facet, probably corresponds to well-known MillerBravais indices (1011) of the same angle (see, e.g., Goldie et al., 1976). The less-frequent, smaller-angle pyramidal face (at $14^{\circ}$ ) is consistent with Miller-Bravais indices 202̄1. Although this facet has not, to our knowledge, been reported in connection with vapor-deposited ice growth, it has been 

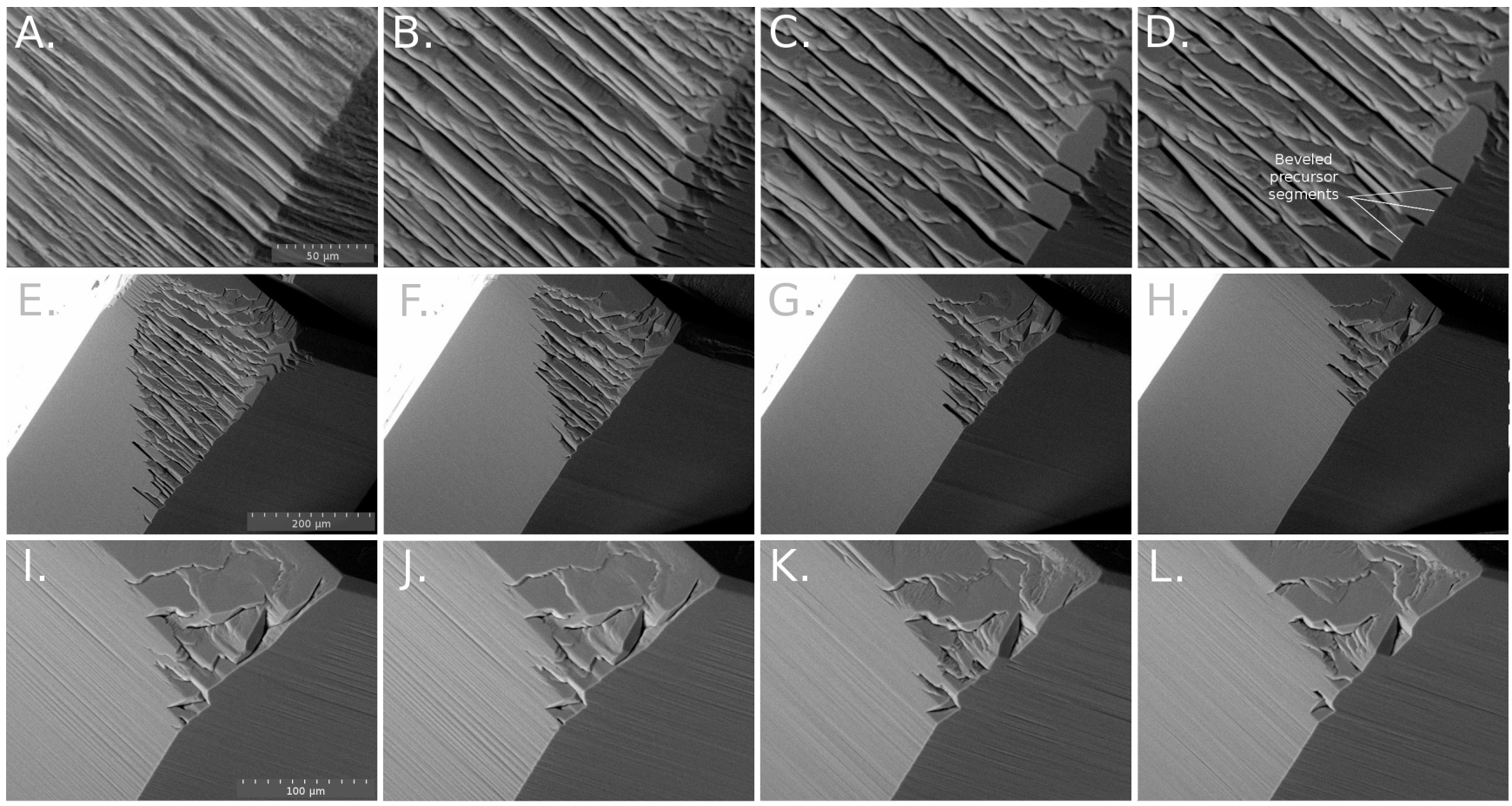

Fig. 6. Re-growth sequences of a previously ablated crystal. Scale bars in the first image of each series apply to all images in the series. (A-D) View of the prismatic edge during initial regrowth of an ablated crystal at intervals of $\sim 45$ seconds. (E-H) Regrowth on the same crystal a few minutes later, at lower magnification, at intervals of $\sim 90 \mathrm{~s}$. (I-L) Final regrowth of the ablated edge, at higher magnification, at intervals of $\sim 45$ seconds.

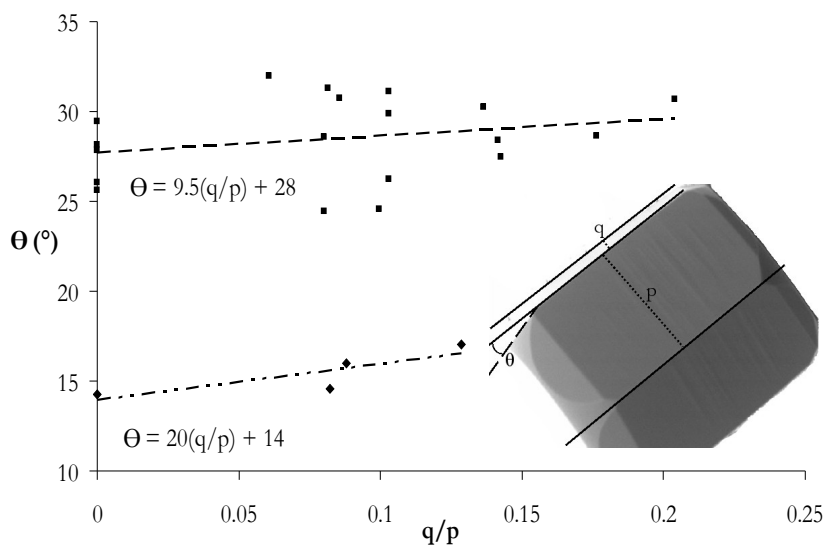

Fig. 7. Detail showing the measurement of the prismatic-topyramidal angle, $\theta$, for a crystal imaged by VP-SEM.

observed in ice crystals grown in aqueous antifreeze solutions derived from fish (Knight et al., 1991; Houston et al., 1998).

In the MD study of Pereyra and Carignano (2009), it was argued that prismatic edges are atomistically robust. The present results reinforce this idea in several ways. Our MD system differs from that of Pereyra and Carignano (2009) in that a different intermolecular potential was used, and the

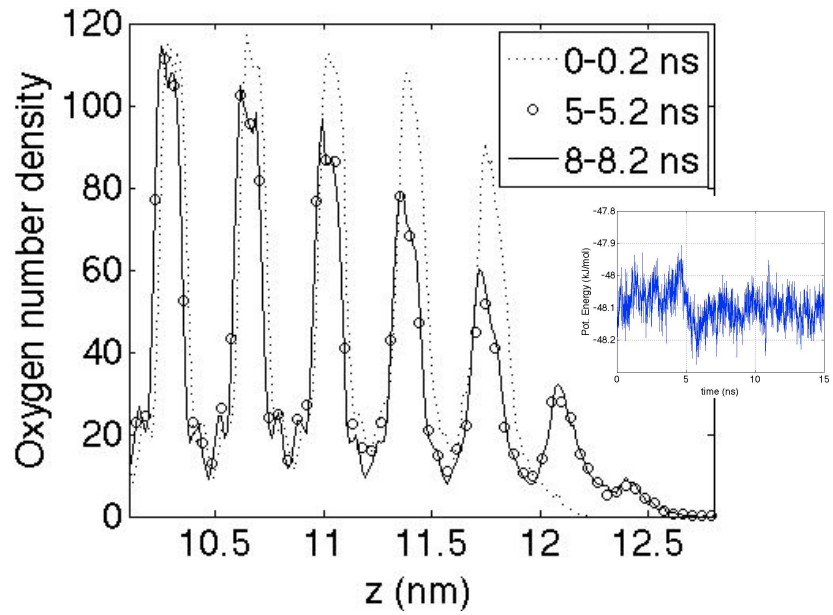

Fig. 8. Integrated number density of oxygen atoms of the freestanding nanocolumn of 8400 water molecules, as a function of distance along the crystallographic c-axis (z), simulated by molecular dynamics. Time $=0$ refers to the moment at which basal surfaces were exposed to vacuum. The inset shows the mean molecular potential energy over time. 


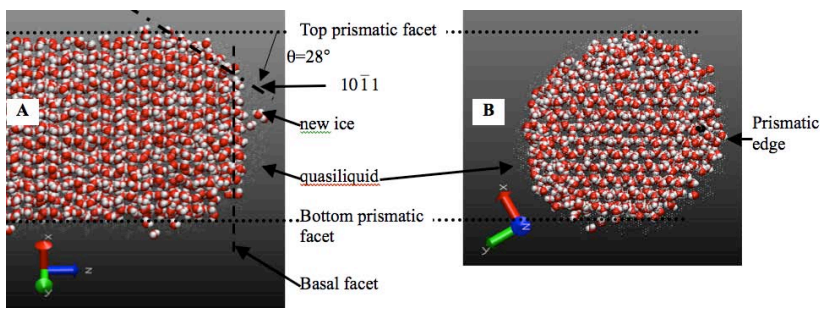

Fig. 9. VMD Representations of the freestanding nanocolumn, $4 \mathrm{~ns}$ after exposure of basal surfaces to vacuum. Molecules rendered as solids were identified as ice-like using a hydrogen bonding criterion described in the text, over the preceeding nanosecond; molecules rendered semi-transparently were identified as quasiliquid. "New ice" refers to molecules that became ice-like after exposing the basal surface to vacuum. (A) View along the $11 \overline{2} 0$, or secondary prismatic facet (Pereyra and Carignano, 2009). (B) Basal view.

nanocolumn was free-standing, but atomistically stable prismatic edges persisted anyway. We have, moreover, found atomistically stable facet edges at the intersections of the pyramidal surface with basal and prismatic facets. The SEM micrographs presented here provide experimental evidence that these edges are also robust at much larger scales than the molecular: the mesoscopic scale is $10^{4}$ times larger than the atomistic.

Do real cirrus ice crystals exhibit surface features such as those seen here? Significant environmental differences exist for ice grown inside the VP-SEM chamber vis-a-vis ice in cirrus clouds: our crystals are grown on a substrate, not as free particles, and the background gas is mostly water vapor at a much lower pressure $(50 \mathrm{~Pa})$ than occurs in cirrus clouds (typically $\sim 200 \mathrm{hPa}$ ), either of which could lead to artifacts. Considering first the role of supersaturation, we note that the surface structures appearing in Figs. 3F and 3G occur at supersaturations on the order of $-25 \%$ to $+25 \%$. Natural cirrus clouds, for comparison, experience a range of supersaturations as high as $150 \%$ and as low as $-50 \%$ (Krämer et al., 2009), although much smaller in-cloud values are more typical. It is important to note that our observations indicate that surface structure is most pronounced near zero supersaturation. Therefore, a relevant in situ analog is the study of Walden et al. (2003) in which falling ice crystals at South Pole Station, imaged after long exposure to near-zero supersaturations, exhibited surface structure of a scale and geometry suggestive of trans-prismatic strands (e.g., Fig. 2c of Walden et al., 2003). Considering the role of pressure, we note that cirrus clouds are believed to experience growth regimes ranging from kinetics-limited growth, to the more efficient diffusion-limited growth (Harrington et al., 2009). The former regime is associated with small values of the ice deposition coefficient, $\alpha_{d}$; recent in situ measurements put its value as low as 0.005 in some instances (MaGee et al., 2006). Within the theoretical framework for vapor-deposited growth on a spherical ice particle (Pruppacher and Klett, 1997), the ice deposition coefficient is inversely proportional to the vapor kinetic length, $l_{d}$ (in the notation of Harrington et al., 2009),

$l_{d}=\frac{4 D_{v}}{\alpha_{d} \bar{v}_{v}}$

where $D_{v}$ is the vapor diffusivity in air and $\bar{v}_{v}$ is the mean speed of water vapor molecules. For typical conditions at the cold stage inside the VP-SEM $\left(T=-45^{\circ} \mathrm{C}, P=50 \mathrm{~Pa}\right)$, Eq. (2) leads to $l_{d} \geq 200 \mu \mathrm{m}$, where the equality corresponds to the maximum theoretical value for the deposition coefficient, $\alpha_{d}=1$. Given that the condition for kinetics-limited growth is $\frac{l_{d}}{r}>1$, where $r$ is the particle radius, we infer that growth of crystals in the VP-SEM is at least significantly kinetics-constrained, and strongly kinetics-limited if $\alpha_{d}$ is significantly smaller than unity. In the latter case, it follows that vapor diffusivity may be expected to have a small influence on surface dynamics. This conclusion follows from a consideration of the Pruppacher-Klett kinetically-corrected vapor growth equation, in which $D_{v}$ is replaced by a kinetically modified diffusivity, $D_{v}^{*}$ (Pruppacher and Klett, 1997). In the strongly kinetics-limited growth regime, $D_{v}^{*}$ is asymptotically independent of $D_{v}$. Thus, although the mean free path of water vapor in the VP-SEM chamber $(\sim 100 \mu \mathrm{m})$ is much greater than that of water vapor near a typical cirrus cloud $(\sim 0.3 \mu \mathrm{m}$; Harrington et al., 2009), the effect on the rate of crystal growth is small. Finally, it is worth mentioning limitations of the Pruppacher-Klett theoretical framework for ice growth that merit further investigation. First, in assuming a spherical surface, it is not clear how the framework might address effects of surface features that lie normal to the radial direction, for example the 5-10 micrometer spacing between features such as transprismatic strands. Second, the framework is meant to apply to growth, not ablation; there is no formal equivalent of a deposition coefficient, for example, when supersaturation is negative. The present work shows, however, that mesoscopic structuring is similar across the growth-to-ablation transition. For these reasons, it is hoped that the present work may offer some insight into the growth and ablation mechanics and mesoscopic structure of cirrus ice.

If it is the case that trans-prismatic strands occur in cirrus clouds at the transition from growth to ablation, it follows that ablating cirrus clouds would be more reflective, gram for gram, than would growing cirrus clouds. The present results would be useful in constructing more realistic parameterizations of the optical scattering properties of cirrus clouds, in as much as the hexagonal symmetry of trans-prismatic strands would scatter light differently than would surface roughness parameterizations currently employed (Yang et al., 2008b). 


\section{Summary}

Trans-prismatic strands represent a form of pronounced mesoscopic roughness on prismatic surfaces of VP-SEMgrown hexagonal ice crystals. This roughness is most prominent at the onset of ablation. During growth, these strands are spaced 5-10 $\mu \mathrm{m}$ apart, and collectively extend over interiors of prismatic facets more than prismatic edges. During ablation, trans-prismatic strands are characterized by a deepening and wider distribution of separations. Prismatic edges are evident and robust under both growth and ablation conditions. Pyramidal facets consistent with Miller-Bravais indices of $10 \overline{1} 1$, and possibly also $20 \overline{2} 1$, are associated with vapor-deposited ice growth under these conditions. Molecular dynamics simulations show that the integrity of facet edges appearing in these crystals has an atomistic analog. While it is argued that these mesoscopic structures may have analogs in cirrus ice under certain conditions, such relevance remains an open question because of the low pressure inside the VP-SEM chamber.

Acknowledgements. We thank A. Vallecorsa and R. Bentson for their technical assistance, and Penny Rowe and three anonymous reviewers for their insightful comments. W. C. P. and R. M. H. were supported by Canfield Research Grants administered through the University of Puget Sound. The authors would like to dedicate this paper to the memory of Stewart Lowther, with whom S. P. N. spent many fruitful hours at an SEM console.

Edited by: T. Garrett

\section{References}

Bailey, M. and Hallett, J.: Growth Rates and Habits of Ice Crystals between $-20^{\circ} \mathrm{C}$ and $-70^{\circ} \mathrm{C}$, J. Atmos. Sci., 61, 514-544, 2004.

Buch, V., Sandler, P., and Sadlej, J.: Simulations of $\mathrm{H}_{2} \mathrm{O}$ Solid, Liquid, and Clusters, with an Emphasis on Ferroelectric Ordering Transition in Hexagonal Ice, J. Phys. Chem. B, 102, 8641-8653, 1998.

Buck, A. L.: New equations for computing vapor pressure and enhancement factor, J. Appl. Meteorol., 20, 1527-1532, 1981.

Carignano, M., Shepson, P., and Szleifer, I.: Molecular dynamics simulations of ice growth from supercooled water, Mol. Phys. 103, 2957-2967, 2005.

Cartwright, J. H. E., Escribano, B., and Sainz-Diaz, C. I.: The mesoscale morphologies of ice films: Porous and biomorphic forms of ice under astrophysical conditions, Astrophys. J., 687, 1406-1414, 2008.

Cross, J. D.: Study of the surface of ice with a scanning electron microscope, in: Physics of Ice: Proceedings of the international symposium on physics of ice, edited by: Riehl, N., Bullemer, B., and Engelhardt, H., Plenum, 81-94, 1968.

Erbe, E. F., Rango, A., Foster, J., Josberger, E. G., Pooley, C., and Wergin, W. P.: Collecting, Shipping, Storing, and Imaging Snow Crystals and Ice Grains With Low-Temperature Scanning Electron Microscopy, Microsc. Res. Tech., 62, 19-32, 2003.
Garrett, T. J.: Observational quantification of the optical properties of cirrus cloud: In Light Scattering Reviews, 3, edited by: Kokhanovsky, P. A., 2008.

Gerber, H., Takano, Y., Garrett, T. J., and Hobbs, P. V.: Nephelometer Measurements of the Asymmetry Parameter, Volume Extinction Coefficient, and Backscatter Ratio in Arctic Clouds, J. Atmos. Sci. 57, 3021-3034, 2000.

Goldie, E. C. W., Meaden, G. T., and White, R.: The concentric halo display of 14 April 1974, Weather, 31, 304-311, 1976.

Gravner, J. and Griffeath, D.: Modeling snow-crystal growth: A three-dimensional mesoscopic approach, Phys. Rev E, 79, 011601-1-18, 2009.

Hallett, J. and Mason, B. J.: The Influence of Temperature and Supersaturation on the Habit of Ice Crystals Grown from the Vapour, Proceedings of the Royal Society of London, Series A, Math. Phys. Sci., 247(1251), 440-453 1958.

Harrington, J. Y., Lamb, D., and Carver, R.: Parameterization of surface kinetic effects for bulk microphysical models: Influences on simulated cirrus dynamics and structure, J. Geophys. Res., 114(D15), D06212, doi:10.1029/2008JD011050, 2009.

Heymsfield, A. J., and Iaquinta, J.: Cirrus crystal terminal velocities, J. Atmos. Sci., 57(7), 916-938, 2000.

Houston, Jr., M. E., Chao, H., Hodges, R. S., Sykes, B. D., Kay, C. M., Sonnichsen, F. D., Loewen, M. C., and Davies, P. L.: Binding of an Oligopeptide to a Specific Plane of Ice, J. Biol. Chem., 273, 11714-11718, 1998.

Humphrey, W., Dalke, A., and Schulten, K.: VMD -Visual Molecular Dynamics, Journal of Molecular Graphics, 14, 33-38, 1996.

Knight, C. A,, Cheng, C. C., and DeVries, A. L.: Adsorption of ahelical antifreeze peptides on specific ice crystal surface planes, Biophys. J., 59, 409-418, 1991.

Krämer, M., Schiller, C., Afchine, A., Bauer, R., Gensch, I., Mangold, A., Schlicht, S., Spelten, N., Sitnikov, N., Borrmann, S., de Reus, M., and Spichtinger, P.: Ice supersaturations and cirrus cloud crystal numbers, Atmos. Chem. Phys., 9, 3505-3522, 2009, http://www.atmos-chem-phys.net/9/3505/2009/.

Lampert, A., Ehrlich, A., Dörnbrack, A., Jourdan, O., Gayet, J.F., Mioche, G., Shcherbakov, V., Ritter, C., and Wendisch, M.: Microphysical and radiative characterization of a subvisible midlevel Arctic ice cloud by airborne observations a case study, Atmos. Chem. Phys., 9, 2647-2661, 2009, http://www.atmos-chem-phys.net/9/2647/2009/.

Libbrecht, K. G.: Explaining the formation of thin ice crystal plates with structure-dependent attachment kinetics, J. Cryst. Growth, 258, 168-175, 2003.

Lindahl, E., Hess, B., and van der Spoel, D.: GROMACS 3.0: A package for molecular simulation and trajectory analysis, J. Mol. Model., 7, 306-317, 2001.

Magee, N., Moyle, A., and Lamb, D.: Experimental determination of the deposition coefficient of small cirruslike crystals near $-50^{\circ} \mathrm{C}$, Geophys. Res. Lett., L17813, doi:10.1029/2006GL026665, 2006.

Nada, H. and van der Eerden, J. P. J. M.: An intermolecular potential model for the simulation of ice and water near the melting point: A six-site model of $\mathrm{H}_{2} \mathrm{O}$, J. Chem. Phys., 118, 7401-7413, 2003.

Neshyba, S. P, Grenfell, T. C., and Warren, S. G.: Representation of a nonspherical ice particle by a collection of independent spheres for scattering and absorption of radiation: 2. Hexag- 
onal columns and plates, J. Geophys. Res., 108(D15), 4448, doi:10.1029/2002JD003302, 2003.

Pereyra, R. G. and Carignano, M. A.: Ice Nanocolumns: A Molecular Dynamics Study, J. Phys. Chem. C., 113, 12699-12705, doi:10.1021/jp903404n, 2009.

Pruppacher, H. R., and Klett, J. D.: Microphysics of Clouds and Precipitation, $2^{\text {nd }}$ Ed., Kluwer Acad., Boston, Mass., USA, 954 pp., 1997.

Rango, A., Wergin, W. P., Erbe, E. F., and Josberger, E. G.: Snow crystal imaging using scanning electron microscopy: III. Glacier ice, snow and biota, Hydrol. Sci. J., 45, 357-375, 2000.

Stephens, G. L., Tsay, S. C., Stackhouse, P. W., and Flatau, P. J.: The relevance of the microphysical and radiative properties of cirrus clouds to climate and climate feedback. J. Atmos. Sci., 47, 1742-1753, 1990.

Ulanowski, Z., Hesse, E., Kaye, P. A., and Baran, A. J.: Light scattering by complex ice-analogue crystals, J. Quant. Spectrosc. Radiat. Transfer, 100, 382-392, 2006.

Wood, S. E., Baker, M. B., and Calhoun D.: New model for the vapor growth of hexagonal ice crystals in the atmosphere, J. Geophys Res., 106, 4845-4870, 2001.

Walden, V. P., Warren, S. G., and Tuttle, E.: Atmospheric Ice Crystals over the Antarctic Plateau in Winter, J. Appl. Meteor., 42, 1391-1405, 2003.
Yang, P. and Liou, K. N.: Single-scattering properties of complex ice crystals in terrestrial atmosphere, Contr. Atmos. Phys. 71, 223-248, 1998.

Yang, P., Wei, H., Huang, H. L., et al.: Scattering and absorption property database for nonspherical ice particles in the nearthrough far-infrared spectral region, Appl. Opt., 44, 5512-5523, 2005.

Yang, P., Zhang, Z., Kattawar, G. W., Warren, S. G., Baum, B. A., Huang, H.-L., Hu, Y. X., Winker, D., and Iaquinta, J.: Effect of Cavities on the Optical Properties of Bullet Rosettes: Implications for Active and Passive Remote Sensing of Ice Cloud Properties, J. Appl. Meteorol. Clim., 47, 2311-2330, 2008 a.

Yang, P., Hong, G., Kattawar, G. W., Minnis, P., and Hu, Y.: Uncertainties Associated With the Surface Texture of Ice Particles in Satellite-Based Retrieval of Cirrus Clouds: Part II-Effect of Particle Surface Roughness on Retrieved Cloud Optical Thickness and Effective Particle Size, IEEE T. Geosci. Remote Sens., 46(7), 1948-1957, 2008b.

Zimmermann, F., Ebert, M., Worringen, A., Schutz, L., and Weinbruch, S.: Environmental scanning electron microscopy (ESEM) as a new technique to determine the ice nucleation capability of individual atmospheric aerosol particles, Atmos. Environ., 41, 8219-8227, 2007. 